\title{
Diabetes self-care practice and disease knowledge in patients with type 2 diabetes mellitus: A cross-sectional analysis with respect to age, educational attainment, income class and ongoing anti-diabetic treatments
}

\author{
Gulbin Seyman Cetinkaya 1, ${ }^{*}$, Fatma Yesim Tan ${ }^{2}$, Haydar Yasa ${ }^{3}$ and Mine Nokay ${ }^{4}$ \\ 1 Department of Internal Medicine, Katip Celebi University Izmir Ataturk Training and Research Hospital, Izmir, Turkey. \\ 2 Department of Internal Medicine, Government Hospital Torball, Izmir, Turkey. \\ ${ }^{3}$ Department of Cardiovascular Surgery, University of Health of Sciences Tepecik Training and Research Hospital, Izmir, \\ Turkey. \\ ${ }^{4}$ Department of Internal Medicine, Antalya Training and Research Hospital, Antalya, Turkey.
}

Magna Scientia Advanced Research and Reviews, 2021, 02(02), 052-069

Publication history: Received on 22 April 2021; revised on 16 June 2021; accepted on 18 June 2021

Article DOI: https://doi.org/10.30574/msarr.2021.2.2.0035

\begin{abstract}
Objective: To evaluate diabetes self-care practice and disease knowledge in type 2 diabetes mellitus (T2D) patients with respect to age, educational attainment, income class and antidiabetic treatments.

Methods: A total of 583 patients with T2D (mean (SD) age: 57.3 (9.5) years, 55.9\% females) were included in this crosssectional study. Data on sociodemographic characteristics, diabetes characteristics (duration, current treatment), diabetes self-care practice [forgetting to take anti-diabetic medication, discontinuation of treatment due to side effects, self-monitoring of blood glucose (SMBG), diabetes education and regular exercise] and disease knowledge (definition and target levels of $\mathrm{HbA1c}$, hypoglycemia symptoms and diabetes-related complications) were recorded.
\end{abstract}

Results: Overall, HbA1c levels were $>8 \%$ in $53.2 \%$ of patients, $38.3 \%$ of patients reported that they had no diabetes education, while at least twice daily SMBG and regular exercise was reported by $27.4 \%$ and $19.7 \%$ of patients, respectively. Lack of knowledge on definition and target levels of HbA1c was noted in up to $65.5 \%$ of patients, while majority of patients reported that they know hypoglycemia symptoms (89.2\%) and diabetes-related complications $(86.4 \%)$. Illiteracy was associated with higher likelihood of treatment discontinuation $(\mathrm{p}<0.001)$ and with lesser likelihood of performing regular exercise (10.3 vs. $32.8 \%, \mathrm{p}<0.001)$. Older patient age, lower educational attainment and lower income level were associated with lesser likelihood of knowing the definition or target levels of HbA1c $(\mathrm{p}<0.001$ for each) and sexual complications of diabetes $(\mathrm{p}<0.001, \mathrm{p}<0.001$ and $\mathrm{p}=0.028$, respectively). Knowing diabetes-related complications were less common in those with lower educational attainment $(p<0.001)$ and lower income level ( $\mathrm{p}=0.010)$, while insulin-naïve patients were less likely to know hypoglycemia symptoms $(\mathrm{p}=0.010)$.

Conclusion: In conclusion, our findings revealed poor glycemic control, low level of knowledge on definition and targets of HbA1c and lack of diabetes education with suboptimal adherence to self-care practice in a considerable percentage of patients. Disease knowledge but not SMBG practice significantly differed with respect to patient age, educational attainment, income class and treatment. Our findings seem to indicate lower disease-related insight among older patients and those with lower educational and income levels, emphasizing the potential role of individualized diabetes education interventions tailored to needs of patients to improve disease knowledge and thus the adherence to self-care practice in $\mathrm{T} 2 \mathrm{D}$ patients.

\footnotetext{
* Corresponding author: Gulbin Seyman Cetinkaya

Department of Internal Medicine, Katip Celebi University Izmir Ataturk Training and Research Hospital, Izmir. 
Keywords: Type 2 diabetes mellitus; Diabetes self-care; Disease knowledge; Diabetes education; Socioeconomic status

\section{Introduction}

Despite novel therapeutics, poor glycemic control has consistently been reported among patients with T2D worldwide $[1,2]$, resulting in an increased risk of microvascular complications, diabetes-related mortality, and all-cause mortality [3].

Diabetes is a self-managed disease necessitating several modifications in health behavior (i.e. dietary change, exercise and medication adherence) to prevent further morbidity and therefore patient understanding, persistence and education in self-care practice are crucial to optimize health outcomes [4-6]. In this regard, identification of diseaserelated knowledge, attitudes and practices of patients with diabetes is considered important to understand the level of acceptance of the disease among patients and patient adherence to treatment and self-care strategies and to develop more effective strategies for patient-centered care [7-12].Importantly, the socioeconomic status, as defined by income and education level, is considered to have significant impact on risk of developing diabetes as well as on the healthrelated behaviors and clinical outcome after diagnosis, with poorer treatment adherence, poorer glycemic control and higher rate of diabetes-related complications in patients with lower educational attainment and lower income levels [13-15]. However, educational attainment is often overlooked in studies addressing efficacy of diabetes interventions among patients with type 2 diabetes mellitus (T2D), despite the risk of disproportionate burden of the increasingly growing disease as well as the likelihood of limited efficacy of interventions in patients with lower levels of education [13,15-17]. Similarly, as a variable often controlled for in the clinical research, the association of patient age with diabetes self-care practice also remains largely unknown, despite it is considered a significant and important factor in the diabetes management $[18,19]$.

This cross-sectional study was therefore designed to evaluate diabetes self-care practice and disease knowledge in diabetic patients with respect to patient age, educational attainment, income class and ongoing anti-diabetic treatment.

\section{Method}

A total of 583 patients with T2D (mean (SD) age: 57.3 (9.5) years, 55.9\% females) who were admitted to our internal medicine outpatient clinics between December 2017 and September 2018 were included in this cross-sectional study. Patients aged $<18$ or $>70$ years, diagnosed with diabetes for less than one year and receiving OAD monotherapy were excluded from the study.

Written informed consent was obtained from each subject following a detailed explanation of the objectives and protocol of the study which was conducted in accordance with the ethical principles stated in the "Declaration of Helsinki" and approved by the institutional ethics committee.

\subsection{Study parameters}

Data on sociodemographic characteristics (age, gender, educational status, income class), hospital characteristics (distance to home, waiting time, quality of doctor-patient relationship), diabetes characteristics (duration, current treatment, glycemic control), diabetes self-care practice (adherence to anti-diabetic treatment, self-monitoring of blood glucose [SMBG] at home, diabetes education, dietary education, regular exercise) and disease knowledge regarding definition and target levels of glycated hemoglobin (HbA1c), hypoglycemia symptoms (headache, sweating, blurred vision, difficulty concentrating) and diabetes-related complications [retinopathy, nephropathy, neuropathy, cardiovascular disease (CVD), sexual dysfunction] were recorded in each patient after completion of the physician visit. The patient-reported data were collected via face-to-face interview method in a separate room by a physician and/or nurse who did not participate in the initial patient examination.

Considering monthly income, the categories were based on Turkey's minimum wage level set by the government, while monetary results were converted by using average $4.8 \mathrm{USD} / \mathrm{TL}$ exchange rates within the study period. Accordingly, income class, based on monthly income, was categorized into lowest $(<2000$ TL [USD 417]), middle (2000-5000 TL [USD 417-1042]), high (5000-10000 TL [USD 1042-2083]) and highest ( $>10000$ TL [ $>$ USD 2083]) income class groups.

Diabetes self-care practice and disease knowledge were evaluated overall as well as with respect to educational attainment, while disease knowledge and SBGM practice were also evaluated with respect to patient age and income class. Knowledge on hypoglycemia symptoms and diabetes-related complications was also analyzed according to type of ongoing anti-diabetic treatments. 


\subsection{Statistical Analysis}

Statistical analysis was made using IBM SPSS Statistics for Windows, version 22.0 (IBM Corp., Armonk, NY). Pearson Chi-square $(\chi 2)$ test was used for the comparison of categorical data. Data were expressed as "mean (standard deviation; SD)" and percent (\%) where appropriate. $\mathrm{p}<0.05$ was considered statistically significant.

\section{Results}

\subsection{Sociodemographic, clinical and hospital-related characteristics}

The mean age was 57.3 years (SD 9.5, 68.3\% aged 45-65 years) and females composed 55.9\% of the study population. Primary education $(60.5 \%)$ and lowest income class (48.9\%) were commonly noted in the study population (Table 1 ).

Diabetes duration was less than 10 years in $56.6 \%$ of patients and the ongoing treatments included OADs in $41.3 \%$ of patients, OADs + insulin in $46.3 \%$ of patients and insulin in $12.3 \%$ of patients. HbA1c levels at the time of study visit were $<7 \%, 7-8 \%$ and $>8 \%$ in $21.4 \%, 25.4 \%$ and $53.2 \%$ of patients, respectively (Table 1 ).

Considering hospital-related characteristics, majority of patients reported no problem related to distance from home to hospital (not far by $85.3 \%$ ), waiting time in the hospital (not long by 88\%) and quality of doctor-patient relationship (very good-moderate by $85.3 \%$ ) (Table 1 ).

Table 1 Sociodemographic, clinical and hospital-related characteristics $(n=583)$

\begin{tabular}{|l|c|}
\hline \multicolumn{1}{|c|}{ Sociodemographic characteristics } \\
\hline Age (year), mean (SD) & $57.3(9.5)$ \\
\hline Age group, $n$ (\%) & \\
\hline $18-45$ year & $61(10.5)$ \\
\hline $46-65$ year & $398(68.3)$ \\
\hline$>65$ year & $124(21.3)$ \\
\hline Gender, $n$ (\%) & \\
\hline Female & $326(55.9)$ \\
\hline Male & $257(44.1)$ \\
\hline Educational status, $n$ (\%) & \\
\hline Illiterate & $87(14.9)$ \\
\hline Primary education & $353(60.5)$ \\
\hline Secondary education & $79(13.6)$ \\
\hline Higher education & $64(11)$ \\
\hline Income class, $n$ (\%) & \\
\hline Lowest & $285(48.9)$ \\
\hline Middle & $242(41.5)$ \\
\hline High & $44(7.5)$ \\
\hline Highest & $12(2.1)$ \\
\hline Disease and treatment characteristics & \\
\hline Diabetes duration, $n(\%)$ & \\
\hline$<5$ year & \\
\hline $6-10$ year & \\
\hline
\end{tabular}




\begin{tabular}{|c|c|}
\hline 11-15 year & $130(22.3)$ \\
\hline$\geq 16$ year & $123(21.1)$ \\
\hline \multicolumn{2}{|l|}{ Current treatment, n (\%) } \\
\hline Oral antidiabetic agents (OADs) & $241(41.3)$ \\
\hline OADs + insulin & $270(46.3)$ \\
\hline Insulin & $72(12.3)$ \\
\hline \multicolumn{2}{|l|}{ HbA1c level, n (\%) } \\
\hline$<7 \%$ & $125(21.4)$ \\
\hline $7-8 \%$ & $148(25.4)$ \\
\hline$>8 \%$ & $310(53.2)$ \\
\hline \multicolumn{2}{|l|}{ Hospital-related variables } \\
\hline \multicolumn{2}{|l|}{ Home to hospital distance, n (\%) } \\
\hline Very close & $202(34.6)$ \\
\hline Close & $170(29.2)$ \\
\hline Moderate & $124(21.3)$ \\
\hline Far & $37(6.3)$ \\
\hline Too far & $50(8.6)$ \\
\hline \multicolumn{2}{|l|}{ Waiting time in the hospital, $\mathrm{n}(\%)$} \\
\hline Very good & $143(24.5)$ \\
\hline Good & $212(36.4)$ \\
\hline Moderate & $158(27.1)$ \\
\hline Poor & $41(7.0)$ \\
\hline Very poor & $29(5.0)$ \\
\hline \multicolumn{2}{|l|}{ Doctor-patient relationship, n (\%) } \\
\hline Very good & $199(34.1)$ \\
\hline Good & $225(38.6)$ \\
\hline Moderate & $73(12.5)$ \\
\hline Poor & $38(6.5)$ \\
\hline Very poor & $48(8.2)$ \\
\hline
\end{tabular}

\subsection{Diabetes self-care practice overall and according to educational status}

Overall, forgetting to take medications was reported by $51.2 \%$ of patients (seldom by $44.9 \%$ ), while treatment discontinuation due to side effects of the drug, initiation of a herbal medicine or fasting during Ramadan was reported by $28.1 \%$ (seldom by $24.0 \%$ ), $14.1 \%$ (seldom by $12.2 \%$ ) and $21.6 \%$ (seldom by $15.1 \%$ ) of patients, respectively (Table 2).

At least twice daily SMBG was reported by $27.4 \%$ of patients, while regular exercise for at least 3 times a week was reported by $19.7 \%$ of patients. In total, $38.3 \%$ of patients reported lack of previous participation in diabetes education, $42.7 \%$ reported to take diabetes education class long time ago and $33.1 \%$ reported to take nurse-led diabetes education. While $68.0 \%$ of patients reported to take dietary education, only $30.4 \%$ confirmed that they adhere to dietary instructions (Table 2). 
Illiteracy was associated with higher likelihood of treatment discontinuation or dose reduction in fasting during Ramadan as compared with primary, secondary and higher education $(19.5 \%$ vs. $4.8 \%, 3.8 \%$ and $1.6 \%$, respectively, $\mathrm{p}<0.001$ ) and with lesser likelihood of performing regular exercise for at least 3 days a week as compared to higher education (10.3 vs. 32.8\%, p<0.001). Illiteracy and primary education were both associated with higher likelihood of never exercising as compared to secondary and higher education (32.2\% and $28.3 \%$ vs. $13.9 \%$ and $10.9 \%$, respectively, $\mathrm{p}<0.001$ ) (Table 2).

No significant difference was noted in type of ongoing anti-diabetic treatments, rates for previous diabetes education and dietary education or SMBG practice according to educational attainment (Table 2).

\subsection{Disease knowledge overall and according to educational status}

Overall, $54.9 \%$ of patients had no knowledge about the definition of HbA1c, and 10.6\% of patients with knowledge on HbA1c reported lack of knowledge regarding target HbA1c levels (Table 3).

Majority of patients reported that they know hypoglycemia symptoms (89.2\%) and diabetes-related complications (86.4\%). The most and least commonly rated hypoglycemia symptoms were heavy sweating (76.3\%) and difficulty concentrating $(44.3 \%)$, while the most and least commonly rated diabetes-related complications were retinopathy $(78.7 \%)$ and sexual dysfunction $(27.1 \%)$, respectively (Table 3$)$.

There was a significant increase in the likelihood of knowing the definition of HbA1c (from 5.7\% for illiteracy to 71.9\% for higher education, $\mathrm{p}<0.001$ ) as well as the target HbA1c levels (from $9.2 \%$ for illiteracy to $84.4 \%$ for higher education, $\mathrm{p}<0.001$ ) with increasing educational level (Table 3).

The percentage of patients reporting heavy sweating (99.2\%, $\mathrm{p}=0.008)$ and difficulty concentrating $(60.9 \%, \mathrm{p}=0.026)$ as hypoglycemia symptoms were significantly higher in the higher education group compared to other educational levels groups (Table 3).

The percentage of patients knowing diabetes-related complications overall $(71.3 \%, \mathrm{p}<0.001)$ and retinopathy $(62.1 \%$, $\mathrm{p}<0.001)$ and nephropathy $(58.6 \%, \mathrm{p}<0.001)$ in particular were significantly lower in the illiteracy group than in other educational level groups. The percentage of patients knowing neuropathy $(31.0 \%$ and $42.8 \%$ vs. $62.8 \%$, p<0.001) were significantly lower in the illiteracy and primary education groups compared to higher education group (Table 3 ).

The percentage of patients knowing CVD (37.9\% and 50.1\% vs. 65.8\% and $73.4 \%, \mathrm{p}<0.001$ ) and sexual dysfunction $(11.5 \%$ and $22.7 \%$ vs. $44.3 \%$ and $51.6 \%$, p < 0.001$)$ were significantly lower in the illiteracy and primary education groups compared to secondary and higher education groups (Table 3).

\subsection{Disease knowledge and SMBG practice according to patient age and income class}

Percentage of patients knowing the definition of HbA1c (54.1\% vs. $23.9 \%$ and $21.8 \%$, respectively, p $<0.001)$ as well as the target HbA1c levels (55.7\% vs. 31.4\% and 32.3\%, respectively, p=0.001) was significantly higher in the $18-45$ year age group than in 46-65 year and $>65$ year age groups (Table 4 ).

Percentage of patients reporting difficulty concentrating as hypoglycemia symptom (63.9\% vs. 39.9\%, p=0.001) and reporting CVD as diabetes-related complication (68.9\% vs. 49.7\%, p=0.017) was significantly higher in the $18-45$ year age group than in 46-65 year group, while the percentage of patients reporting sexual dysfunction was significantly higher in the 18-45 year age group than in 46-65 year and $>65$ year age groups (52.5\% vs. 26.6\% and 16.1\%, respectively, $\mathrm{p}<0.001$ ) (Table 4 ).

The lowest income class was associated with lesser likelihood of knowing the definition of HbA1c (vs. middle and high income classes, $\mathrm{p}<0.001$ ), the diabetes-related complications overall (vs. middle income class, $\mathrm{p}=0.010$ ) and retinopathy (vs. middle income class, $\mathrm{p}=0.029$ and sexual dysfunction (vs. highest income class, $\mathrm{p}=0.028$ ), in particular (Table 4).

No significant difference was noted in SMBG practice according to patient age or income class (Table 4). 
Table 2 Diabetes self-care practice overall and according to educational status

\begin{tabular}{|c|c|c|c|c|c|c|}
\hline & \multirow[b]{2}{*}{$\begin{array}{l}\text { Total } \\
(n=583)\end{array}$} & \multicolumn{5}{|c|}{ Educational status } \\
\hline & & $\begin{array}{l}\text { Illiterate } \\
(n=87)\end{array}$ & $\begin{array}{l}\text { Primary education } \\
(n=353)\end{array}$ & $\begin{array}{l}\text { Secondary } \\
\text { education }(n=79)\end{array}$ & $\begin{array}{l}\text { Higher } \\
\text { education } \\
(n=64)\end{array}$ & p value \\
\hline \multicolumn{7}{|l|}{ Diabetes self-care practice } \\
\hline \multicolumn{7}{|l|}{ Current treatment, n (\%) } \\
\hline Oral antidiabetic agents (OADs) & $241(41.3)$ & $42(48.3)$ & $138(39.1)$ & $34(43)$ & $27(42.2)$ & \multirow{3}{*}{0.619} \\
\hline OADs + insulin & $270(46.3)$ & $36(41.4)$ & $172(48.7)$ & $36(45.6)$ & $26(40.6)$ & \\
\hline Insulin & $72(12.3)$ & $9(10.3)$ & $43(12.2)$ & $9(11.4)$ & $11(17.2)$ & \\
\hline \multicolumn{7}{|l|}{ Forgetting to take medications } \\
\hline Seldom & $262(44.9)$ & $46(52.9)$ & $150(42.5)$ & $38(48.1)$ & $28(43.8)$ & \multirow{3}{*}{0.628} \\
\hline Often & $37(6.3)$ & $4(4.6)$ & $26(7.4)$ & $4(5.1)$ & $3(4.7)$ & \\
\hline Never & $284(48.7)$ & $37(42.5)$ & $177(50.1)$ & $37(46.8)$ & $33(51.6)$ & \\
\hline \multicolumn{7}{|c|}{ Discontinuation of treatment due to side effects } \\
\hline Seldom & $140(24.0)$ & $25(28.7)$ & $82(23.2)$ & $19(24.1)$ & $14(21.9)$ & \multirow{3}{*}{0.177} \\
\hline Often & $24(4.1)$ & $8(9.2)$ & $12(3.4)$ & $2(2.5)$ & $2(3.1)$ & \\
\hline Never & $419(71.9)$ & $54(62.1)$ & $259(73.4)$ & $58(73.4)$ & $48(75)$ & \\
\hline \multicolumn{7}{|c|}{$\begin{array}{l}\text { Discontinuation of treatment to start herbal } \\
\text { medicine }\end{array}$} \\
\hline Seldom & $71(12.2)$ & $15(17.2)$ & $40(11.3)$ & $11(13.9)$ & $5(7.8)$ & \multirow{3}{*}{0.507} \\
\hline Often & $11(1.9)$ & $3(3.4)$ & $6(1.7)$ & $1(1.3)$ & $1(1.6)$ & \\
\hline Never & $501(85.9)$ & $69(79.3)$ & $307(87)$ & $67(84.8)$ & $58(90.6)$ & \\
\hline \multicolumn{7}{|c|}{$\begin{array}{l}\text { Discontinuation/ dose reduction in fasting during } \\
\text { Ramadan }\end{array}$} \\
\hline Seldom & $88(15.1)$ & $17(19.5) \mathrm{a}$ & $54(15.3) \mathrm{a}$ & $11(13.9) \mathrm{a}$ & $6(9.5) \mathrm{a}$ & \multirow{3}{*}{$<0.001$} \\
\hline Often & $38(6.5)$ & $17(19.5) \mathrm{a}$ & $17(4.8) \mathrm{b}$ & $3(3.8) b$ & $1(1.6) b$ & \\
\hline Never & $456(78.4)$ & $53(60.9) \mathrm{a}$ & $282(79.9) \mathrm{b}$ & $65(82.3) b$ & $56(88.9) \mathrm{b}$ & \\
\hline \multicolumn{7}{|l|}{ SMBG at home (last 3 months) } \\
\hline None & $104(17.8)$ & $19(21.8)$ & $67(19)$ & $7(8.9)$ & $11(17.2)$ & 0.348 \\
\hline
\end{tabular}




\begin{tabular}{|c|c|c|c|c|c|c|}
\hline Sometimes & $319(54.7)$ & $42(48.3)$ & $194(55)$ & $49(62)$ & $34(53.1)$ & \\
\hline At least twice daily & $160(27.4)$ & $26(29.9)$ & $92(26.1)$ & $23(29.1)$ & $19(29.7)$ & \\
\hline \multicolumn{7}{|c|}{ Participation in diabetes education } \\
\hline Never & $223(38.3)$ & $44(50.6)$ & $127(36)$ & $31(39.2)$ & $21(32.8)$ & \multirow{4}{*}{0.132} \\
\hline A long time ago & $249(42.7)$ & $32(36.8)$ & $152(43.1)$ & $35(44.3)$ & $30(46.9)$ & \\
\hline Within the past 3 months & $78(13.4)$ & $9(10.3)$ & $55(15.6)$ & $8(10.1)$ & $6(9.4)$ & \\
\hline Continuous & $33(5.7)$ & $2(2.3)$ & $19(5.4)$ & $5(6.3)$ & $7(10.9)$ & \\
\hline \multicolumn{7}{|l|}{ Diabetes education by } \\
\hline Physician & $85(14.6)$ & $9(10.3)$ & $47(13.3)$ & $14(17.7)$ & $15(23.4)$ & \multirow{5}{*}{$\mathrm{N} / \mathrm{A}$} \\
\hline Diabetes nurse & 193(33.1) & $23(26.4)$ & $120(34)$ & $25(31.6)$ & $25(39.1)$ & \\
\hline Ward nurse & $27(4.6)$ & $4(4.6)$ & $18(5.1)$ & $4(5.1)$ & $1(1.6)$ & \\
\hline Pen trainer & $45(7.7)$ & $6(6.9)$ & $33(9.3)$ & $5(6.3)$ & $1(1.6)$ & \\
\hline Pharmacist & $10(1.7)$ & $1(1.1)$ & $8(2.3)$ & $0(0)$ & $1(1.6)$ & \\
\hline \multicolumn{7}{|l|}{ Dietary education, $\mathrm{n}(\%)$} \\
\hline Yes, but not adhere & $219(37.6)$ & $36(41.4)$ & $133(37.7)$ & $29(36.7)$ & $21(32.8)$ & \multirow{3}{*}{0.574} \\
\hline Yes and adhere & $177(30.4)$ & $21(24.1)$ & $114(32.3)$ & $20(25.3)$ & $22(34.4)$ & \\
\hline No & $187(32.1)$ & $30(34.5)$ & $106(30)$ & $30(38)$ & $21(32.8)$ & \\
\hline \multicolumn{7}{|l|}{ Regular exercise, $\mathrm{n}(\%)$} \\
\hline No due to comorbidity & $69(11.8)$ & $19(21.8) \mathrm{a}$ & 43(12.2) a.b & $3(3.8) b$ & $4(6.3)$ a.b & \multirow{4}{*}{$<0.001$} \\
\hline Never & $146(25)$ & $28(32.2) \mathrm{a}$ & $100(28.3) \mathrm{a}$ & $11(13.9) \mathrm{b}$ & $7(10.9) b$ & \\
\hline Sometimes & $253(43.4)$ & $31(35.6) \mathrm{a}$ & $144(40.8) \mathrm{a}$ & $46(58.2) b$ & $32(50) a . b$ & \\
\hline$\geq 3$ times a week & $115(19.7)$ & $9(10.3) \mathrm{a}$ & 66(18.7) a.b & $19(24.1)$ a.b & $21(32.8) \mathrm{b}$ & \\
\hline
\end{tabular}

SMBG: Self-monitoring of blood glucose; NA: Not applicable. Pearson chi-square test. For the variables in the same row, statistically significant difference exists only between those marked with different lowercase letters 
Table 3 Disease knowledge overall and according to educational status

\begin{tabular}{|c|c|c|c|c|c|c|c|}
\hline & & \multirow[b]{2}{*}{$\begin{array}{l}\text { Total } \\
(n=583)\end{array}$} & \multicolumn{5}{|c|}{ Educational status } \\
\hline & & & $\begin{array}{l}\text { Illiterate } \\
(n=87)\end{array}$ & $\begin{array}{l}\text { Primary education } \\
(n=353)\end{array}$ & $\begin{array}{l}\text { Secondary education } \\
(n=79)\end{array}$ & $\begin{array}{l}\text { Higher } \\
\text { education } \\
(n=64)\end{array}$ & $\begin{array}{l}\mathbf{p} \\
\text { value }\end{array}$ \\
\hline \multicolumn{8}{|c|}{ Knowledge on definition of HbA1c, n (\%) } \\
\hline Not know & & $320(54.9)$ & $70(80.5)^{\mathrm{a}}$ & $208(58.9)^{b}$ & $33(41.8)^{c}$ & $9(14.1)^{d}$ & \multirow{3}{*}{$<0.001$} \\
\hline Yes & & $155(26.6)$ & $5(5.7)^{\mathrm{a}}$ & $74(21.0)^{b}$ & $30(38.0)^{c}$ & $46(71.9)^{\mathrm{d}}$ & \\
\hline Not remember & & $108(18.5)$ & $12(13.8)^{\mathrm{a}}$ & $71\left(20.1^{\mathrm{a}}\right.$ & $16(20.3)^{\mathrm{a}}$ & $9(14.1)^{\mathrm{a}}$ & \\
\hline \multicolumn{8}{|c|}{ Knowledge on target HbA1c level, n (\%) } \\
\hline Not know & & $62(10.6)$ & $9(10.3)^{\mathrm{a}}$ & $42(11.9)^{\mathrm{a}}$ & $10(12.7)^{a}$ & $1(1.6)^{\mathrm{a}}$ & \multirow{4}{*}{$<0.001$} \\
\hline$<7 \%$ & & $199(34.1)$ & $8(9.2)^{\mathrm{a}}$ & $101(28.6)^{b}$ & $36(45.6)^{c}$ & $54(84.4)^{\mathrm{d}}$ & \\
\hline$>7 \%$ & & $2(0.3)$ & $0(0)^{\mathrm{a}}$ & $2(0.6)^{a}$ & $0(0)^{\mathrm{a}}$ & $0(0)^{\mathrm{a}}$ & \\
\hline No knowledge on HbA1c & & $320(54.9)$ & $70(80.5)^{a}$ & $208(58.9)^{b}$ & $33(41.8)^{c}$ & $9(14.1)^{\mathrm{d}}$ & \\
\hline \multicolumn{8}{|c|}{ Knowledge on hypoglycemia symptoms, n (\%) } \\
\hline Yes & & $520(89.2)$ & $74(85.1)$ & $311(88.1)$ & $73(92.4)$ & $62(96.9)$ & \multirow{2}{*}{0.081} \\
\hline No & & $63(10.8)$ & $13(14.9)$ & $42(11.9)$ & $6(7.6)$ & $2(3.1)$ & \\
\hline \multirow[b]{2}{*}{ Headache } & No & $322(55.2)$ & $39(44.8)$ & $205(58.1)$ & $45(57.0)$ & $33(51.6)$ & \multirow{2}{*}{0.144} \\
\hline & Yes & $261(44.8)$ & $48(55.2)$ & $148(41.9)$ & $34(43.0)$ & $31(48.4)$ & \\
\hline \multirow[b]{2}{*}{ Heavy sweating } & No & $138(23.7)$ & $27(31.0)^{\mathrm{a}}$ & $88(24.9)^{\mathrm{a}}$ & $18(22.8)^{a}$ & $5(7.8)^{b}$ & \multirow{2}{*}{0.008} \\
\hline & Yes & $445(76.3)$ & $60(69.0)^{\mathrm{a}}$ & $265(75.1)^{\mathrm{a}}$ & $61(77.2)^{\mathrm{a}}$ & $59(92.2)^{\mathrm{b}}$ & \\
\hline \multirow[b]{2}{*}{ Blurred vision } & No & $260(44.6)$ & $37(42.5)$ & $155(43.9)$ & $44(55.7)$ & $24(37.5)$ & \multirow{2}{*}{0.141} \\
\hline & Yes & $323(55.4)$ & $50(57.5)$ & $198(56.1)$ & $35(44.3)$ & $40(62.5)$ & \\
\hline \multirow[t]{2}{*}{ Difficulty concentrating } & No & $325(55.7)$ & $50(57.5)^{\mathrm{a}}$ & $200(56.7)^{\mathrm{a}}$ & $50(63.3)^{\mathrm{a}}$ & $25(39.1)^{b}$ & \multirow{2}{*}{0.026} \\
\hline & Yes & $258(44.3)$ & $37(42.5)^{\mathrm{a}}$ & $153(43.3)^{\mathrm{a}}$ & $29(36.7)^{\mathrm{a}}$ & $39(60.9)^{b}$ & \\
\hline
\end{tabular}




\begin{tabular}{|c|c|c|c|c|c|c|c|}
\hline \multicolumn{8}{|c|}{ Knowledge on diabetes-related complications, n (\%) } \\
\hline \multicolumn{2}{|l|}{ Yes } & $504(86.4)$ & $62(71.3)^{a}$ & $309(87.5)^{\mathrm{b}}$ & $71(89.9)^{\mathrm{b}}$ & $62(96.9)^{\mathrm{b}}$ & \multirow{2}{*}{$<0.001$} \\
\hline \multicolumn{2}{|l|}{ No } & $79(13.6)$ & $25(28.7)^{\mathrm{a}}$ & $44(12.5)^{\mathrm{b}}$ & $8(10.1)^{b}$ & $2(3.1)^{b}$ & \\
\hline \multirow[b]{2}{*}{ Retinopathy } & No & $124(21.3)$ & $33(37.9)^{\mathrm{a}}$ & $67(19)^{b}$ & $16(20.3)^{b}$ & $8(12.5)^{b}$ & \multirow{2}{*}{$<0.001$} \\
\hline & Yes & $459(78.7)$ & $54(62.1)^{\mathrm{a}}$ & $286(81)^{\mathrm{b}}$ & $63(79.7)^{b}$ & $56(87.5)^{b}$ & \\
\hline \multirow[b]{2}{*}{ Nephropathy } & No & $146(25)$ & $36(41.4)^{\mathrm{a}}$ & $85(24.1)^{b}$ & $19(24.1)^{b}$ & $6(9.4)^{\mathrm{b}}$ & \multirow{2}{*}{$<0.001$} \\
\hline & Yes & $437(75.0)$ & $51(58.6)^{\mathrm{a}}$ & $268(75.9)^{\mathrm{b}}$ & $60(75.9)^{\mathrm{b}}$ & $58(90.6)^{\mathrm{b}}$ & \\
\hline \multirow[b]{2}{*}{ Neuropathy } & No & $322(55.2)$ & $60(69)^{\mathrm{a}}$ & $202(57.2)^{\mathrm{a}}$ & $40(50.6)^{a . b}$ & $20(31.3)^{b}$ & \multirow{2}{*}{$<0.001$} \\
\hline & Yes & $261(44.8)$ & $27(31.0)^{\mathrm{a}}$ & $151(42.8)^{\mathrm{a}}$ & $39(49.4)^{a . b}$ & $44(68.8)^{b}$ & \\
\hline \multirow[b]{2}{*}{ Cardiovascular disease } & No & $274(47.0)$ & $54(62.1)^{\mathrm{a}}$ & $176(49.9)^{\mathrm{a}}$ & $27(34.2)^{\mathrm{b}}$ & $17(26.6)^{b}$ & \multirow{2}{*}{$<0.001$} \\
\hline & Yes & $309(53.0)$ & $33(37.9)^{\mathrm{a}}$ & $177(50.1)^{\mathrm{a}}$ & $52(65.8)^{b}$ & $47(73.4)^{b}$ & \\
\hline \multirow[b]{2}{*}{ Sexual dysfunction } & No & $425(72.9)$ & $77(88.5)^{\mathrm{a}}$ & $273(77.3)^{\mathrm{a}}$ & $44(55.7)^{\mathrm{b}}$ & $31(48.4)^{b}$ & \multirow{2}{*}{$<0.001$} \\
\hline & Yes & $158(27.1)$ & $10(11.5)^{\mathrm{a}}$ & $80(22.7)^{\mathrm{a}}$ & $35(44.3)^{b}$ & $33(51.6)^{b}$ & \\
\hline
\end{tabular}

Pearson chi-square test. For the variables in the same row, statistically significant difference exists only between those marked with different lowercase letters 
Table 4 Disease knowledge and SMBG practice according to patient age and income class

\begin{tabular}{|c|c|c|c|c|c|c|c|c|c|c|}
\hline & & \multicolumn{4}{|l|}{ Patient age } & \multicolumn{5}{|c|}{ Income class } \\
\hline & & $\begin{array}{l}\text { 18-45 year } \\
\text { (n:61) }\end{array}$ & $\begin{array}{l}46-65 \\
\text { year } \\
(n: 398)\end{array}$ & $\begin{array}{l}>65 \text { year } \\
(n: 124)\end{array}$ & p value & $\begin{array}{l}\text { Lowest } \\
(n=285)\end{array}$ & $\begin{array}{l}\text { Middle } \\
(n=242)\end{array}$ & $\begin{array}{l}\text { High } \\
(n=44)\end{array}$ & $\begin{array}{l}\text { Highest } \\
(n=12)\end{array}$ & p value \\
\hline \multicolumn{11}{|c|}{ Knowledge on definition of HbA1c, n (\%) } \\
\hline \multicolumn{2}{|l|}{ Not know } & $19(31.1)^{\mathrm{a}}$ & $225(56.5)^{b}$ & $76(61.3)^{b}$ & \multirow[t]{3}{*}{$<0.001$} & $188(66.0)^{\mathrm{a}}$ & $116(47.9)^{\mathrm{b}}$ & $11(25.0)^{c}$ & $5(41.7)^{\text {a.b.c }}$ & \multirow{3}{*}{$<0.001$} \\
\hline \multicolumn{2}{|l|}{ Yes } & $33(54.1)^{\mathrm{a}}$ & $95(23.9)^{\mathrm{b}}$ & $27(21.8)^{\mathrm{b}}$ & & $48(16.8)^{a}$ & $78(32.2)^{\mathrm{b}}$ & $25(56.8)^{c}$ & $4(33.3)^{\text {a.b.c }}$ & \\
\hline \multicolumn{2}{|l|}{ Not remember } & $9(14.8)^{a}$ & $78(19.6)^{\mathrm{a}}$ & $21(16.9)^{a}$ & & $49(17.2)^{\mathrm{a}}$ & $48(19.8)^{\mathrm{a}}$ & $8(18.2)^{a}$ & $3(25)^{\mathrm{a}}$ & \\
\hline \multicolumn{11}{|c|}{ Knowledge on target HbA1c level, n (\%) } \\
\hline \multicolumn{2}{|l|}{ Not know } & $7(11.5)^{\mathrm{a}}$ & $47(11.8)^{\mathrm{a}}$ & $8(6.5)^{a}$ & \multirow[t]{4}{*}{0.001} & $33(11.6)$ & $25(10.3)$ & $3(6.8)$ & $1(8.3)$ & \multirow{4}{*}{$\mathrm{N} / \mathrm{A}$} \\
\hline \multicolumn{2}{|l|}{$<7 \%$} & $34(55.7)^{\mathrm{a}}$ & $125(31.4)^{\mathrm{b}}$ & $40(32.3)^{b}$ & & $63(22.1)$ & $100(41.3)$ & $30(68.2)$ & $6(50)$ & \\
\hline \multicolumn{2}{|l|}{$>7 \%$} & $1(1.6)^{a}$ & $1(0.3)^{\mathrm{a}}$ & $0(0)^{\mathrm{a}}$ & & $1(0.4)$ & $1(0.4)$ & $0(0)$ & $0(0)$ & \\
\hline \multicolumn{2}{|l|}{ No knowledge on HbA1c } & $19(31.1)^{\mathrm{a}}$ & $225(56.5)^{b}$ & $76(61.3)^{b}$ & & $188(66)$ & $116(47.9)$ & $11(25)$ & $5(41.7)$ & \\
\hline \multicolumn{11}{|c|}{ SMBG at home (last 3 months) } \\
\hline \multicolumn{2}{|l|}{ None } & $10(16.4)$ & $75(18.8)$ & $19(15.3)$ & \multirow[t]{3}{*}{0.352} & $61(21.4)$ & $33(13.6)$ & $8(18.2)$ & $2(16.7)$ & \multirow{3}{*}{0.125} \\
\hline \multicolumn{2}{|l|}{ Sometimes } & $35(57.4)$ & $222(55.8)$ & $62(50)$ & & $140(49.1)$ & $145(59.9)$ & $28(63.6)$ & $6(50)$ & \\
\hline \multicolumn{2}{|l|}{ At least twice daily } & $16(26.2)$ & $101(25.4)$ & $43(34.7)$ & & $84(29.5)$ & $64(26.4)$ & $8(18.2)$ & $4(33.3)$ & \\
\hline \multicolumn{11}{|c|}{$\begin{array}{l}\text { Knowledge on hypoglycemia symptoms, } \\
\text { n(\%) }\end{array}$} \\
\hline \multicolumn{2}{|l|}{ Yes } & $56(91.8)$ & $352(88.4)$ & $112(90.3)$ & \multirow{2}{*}{0.661} & $251(88.1)$ & $216(89.3)$ & $43(97.7)$ & $10(83.3)$ & \multirow{2}{*}{0.248} \\
\hline \multicolumn{2}{|l|}{ No } & $5(8.2)$ & $46(11.6)$ & $12(9.7)$ & & $34(11.9)$ & $26(10.7)$ & $1(2.3)$ & $2(16.7)$ & \\
\hline \multirow[b]{2}{*}{ Headache } & No & $26(42.6)$ & $223(56)$ & $73(58.9)$ & \multirow[t]{2}{*}{0.096} & $153(53.7)$ & $136(56.2)$ & $26(59.1)$ & $7(58.3)$ & \multirow{2}{*}{0.878} \\
\hline & Yes & $35(57.4)$ & $175(44)$ & $51(41.1)$ & & $132(46.3)$ & $106(43.8)$ & $18(40.9)$ & $5(41.7)$ & \\
\hline \multirow[b]{2}{*}{ Heavy sweating } & No & $10(16.4)$ & $105(26.4)$ & $23(18.5)$ & \multirow[t]{2}{*}{0.074} & $71(24.9)$ & $59(24.4)$ & $4(9.1)$ & $4(33.3)$ & \multirow{2}{*}{0.107} \\
\hline & Yes & $51(83.6)$ & $293(73.6)$ & $101(81.5)$ & & $214(75.1)$ & $183(75.6)$ & $40(90.9)$ & $8(66.7)$ & \\
\hline
\end{tabular}




\begin{tabular}{|c|c|c|c|c|c|c|c|c|c|c|}
\hline \multirow[b]{2}{*}{ Blurred vision } & No & $19(31.1)$ & $185(46.5)$ & $56(45.2)$ & \multirow[t]{2}{*}{0.080} & $114(40)$ & $120(49.6)$ & $19(43.2)$ & $7(58.3)$ & \multirow{2}{*}{0.120} \\
\hline & Yes & $42(68.9)$ & $213(53.5)$ & $68(54.8)$ & & $171(60)$ & $122(50.4)$ & $25(56.8)$ & $5(41.7)$ & \\
\hline \multirow[b]{2}{*}{ Difficulty concentrating } & No & $22(36.1)^{a}$ & $239(60.1)^{\mathrm{b}}$ & $64(51.6)^{a . b}$ & \multirow[t]{2}{*}{0.001} & $159(55.8)$ & $141(58.3)$ & $18(40.9)$ & $7(58.3)$ & \multirow{2}{*}{0.205} \\
\hline & Yes & $39(63.9)^{\mathrm{a}}$ & $159(39.9)^{b}$ & $60(48.4)^{a . b}$ & & $126(44.2)$ & $101(41.7)$ & $26(59.1)$ & $5(41.7)$ & \\
\hline \multicolumn{11}{|c|}{$\begin{array}{lcc}\text { Knowledge } & \text { on } & \text { diabetes-related } \\
\text { complications, } & \text { n (\%) }\end{array}$} \\
\hline \multicolumn{2}{|l|}{ Yes } & $54(88.5)$ & $341(85.7)$ & $109(87.9)$ & \multirow[t]{2}{*}{0.723} & $233(81.8)^{\mathrm{a}}$ & $218(90.1)^{b}$ & $42(95.5)^{a . b}$ & $11(91.7)^{a . b}$ & \multirow{2}{*}{0.010} \\
\hline \multicolumn{2}{|l|}{ No } & $7(11.5)$ & $57(14.3)$ & $15(12.1)$ & & $52(18.2)^{\mathrm{a}}$ & $24(9.9)^{\mathrm{b}}$ & $2(4.5)^{a . b}$ & $1(8.3)^{a . b}$ & \\
\hline \multirow{2}{*}{ Retinopathy } & No & $13(21.3)$ & $86(21.6)$ & $25(20.2)$ & \multirow[t]{2}{*}{0.943} & $75(26.3)^{a}$ & $40(16.5)^{b}$ & $8(18.2)^{a . b}$ & $1(8.3)^{a . b}$ & \multirow{2}{*}{0.029} \\
\hline & Yes & $48(78.7)$ & $312(78.4)$ & $99(79.8)$ & & $210(73.7)^{\mathrm{a}}$ & $202(83.5)^{b}$ & $36(81.8)^{a . b}$ & $11(91.7)^{a . b}$ & \\
\hline \multirow{2}{*}{ Nephropathy } & No & $10(16.4)$ & $109(27.4)$ & $27(21.8)$ & \multirow[t]{2}{*}{0.116} & $84(29.5)$ & $49(20.2)$ & $9(20.5)$ & $4(33.3)$ & \multirow{2}{*}{0.076} \\
\hline & Yes & $51(83.6)$ & $289(72.6)$ & $97(78.2)$ & & $201(70.5)$ & 193(79.8) & $35(79.5)$ & $8(66.7)$ & \\
\hline \multirow[t]{2}{*}{ Neuropathy } & No & $28(45.9)$ & $229(57.5)$ & $65(52.4)$ & \multirow[t]{2}{*}{0.183} & $170(59.6)$ & $127(52.5)$ & $18(40.9)$ & $7(58.3)$ & \multirow{2}{*}{0.083} \\
\hline & Yes & $33(54.1)$ & $169(42.5)$ & $59(47.6)$ & & $115(40.4)$ & $115(47.5)$ & $26(59.1)$ & $5(41.7)$ & \\
\hline \multirow[b]{2}{*}{ CVD } & No & $19(31.1)^{\mathrm{a}}$ & $200(50.3)^{\mathrm{b}}$ & $55(44.4)^{a . b}$ & \multirow[t]{2}{*}{0.017} & $147(51.6)$ & $105(43.4)$ & $17(38.6)$ & $5(41.7)$ & \multirow{2}{*}{0.169} \\
\hline & Yes & $42(68.9)^{a}$ & $198(49.7)^{b}$ & $69(55.6)^{\text {a.b }}$ & & $138(48.4)$ & $137(56.6)$ & $27(61.4)$ & $7(58.3)$ & \\
\hline \multirow{2}{*}{ Sexual dysfunction } & No & $29(47.5)^{\mathrm{a}}$ & $292(73.4)^{b}$ & $104(83.9)^{\mathrm{b}}$ & \multirow[t]{2}{*}{$<0.001$} & $222(77.9)^{\mathrm{a}}$ & $167(69)^{a . b}$ & $30(68.2)^{a . b}$ & $6(50)^{\mathrm{b}}$ & \multirow{2}{*}{0.028} \\
\hline & Yes & $32(52.5)^{\mathrm{a}}$ & $106(26.6)^{\mathrm{b}}$ & $20(16.1)^{b}$ & & $63(22.1)^{\mathrm{a}}$ & $75(31)^{a . b}$ & $14(31.8)^{a . b}$ & $6(50)^{b}$ & \\
\hline
\end{tabular}

CVD: Cardiovascular disease; SMBG: Self-monitoring of blood glucose; NA: Not applicable. Pearson chi-square test. For the variables in the same row, statistically significant difference exists only between those marked with different lowercase letter. 
Table 5 Knowledge on hypoglycemia symptoms and diabetes-related complications according to ongoing anti-diabetic treatments

\begin{tabular}{|c|c|c|c|c|c|}
\hline & & \multicolumn{4}{|c|}{ Current treatment } \\
\hline & & $\begin{array}{l}\text { OADs } \\
(\mathrm{n}=241)\end{array}$ & $\begin{array}{l}\text { OADs + insulin } \\
(n=270)\end{array}$ & $\begin{array}{l}\text { Insulin } \\
(\mathrm{n}=72)\end{array}$ & p value \\
\hline \multicolumn{6}{|c|}{ Knowledge on hypoglycemia symptoms, n (\%) } \\
\hline \multicolumn{2}{|c|}{ Yes } & $204(84.6)^{\mathrm{a}}$ & $251(93.0)^{\mathrm{b}}$ & $65(90.3)^{a . b}$ & 0.010 \\
\hline \multicolumn{2}{|l|}{ No } & $37(15.4)^{\mathrm{a}}$ & $19(7)^{\mathrm{b}}$ & $7(9.7)^{\mathrm{a} . \mathrm{b}}$ & \\
\hline \multirow[b]{2}{*}{ Headache } & No & $123(51)$ & $161(59.6)$ & $38(52.8)$ & \multirow{2}{*}{0.135} \\
\hline & Yes & $118(49)$ & $109(40.4)$ & $34(47.2)$ & \\
\hline \multirow[b]{2}{*}{ Heavy sweating } & No & $80(33.2)^{\mathrm{a}}$ & $43(15.9)^{\mathrm{b}}$ & $15(20.8)^{a . b}$ & \multirow{2}{*}{$<0.001$} \\
\hline & Yes & $161(66.8)^{\mathrm{a}}$ & $227(84.1)^{\mathrm{b}}$ & $57(79.2)^{\text {a.b }}$ & \\
\hline \multirow[b]{2}{*}{ Blurred vision } & No & $127(52.7)^{\mathrm{a}}$ & $105(38.9)^{\mathrm{b}}$ & $28(38.9)^{\mathrm{b}}$ & \multirow{2}{*}{0.004} \\
\hline & Yes & $114(47.3)^{\mathrm{a}}$ & $165(61.1)^{b}$ & $44(61.1)^{\mathrm{b}}$ & \\
\hline \multirow[b]{2}{*}{ Difficulty concentrating } & No & $154(63.9)^{\mathrm{a}}$ & $138(51.1)^{\mathrm{b}}$ & $33(45.8)^{\mathrm{b}}$ & \multirow{2}{*}{0.003} \\
\hline & Yes & $87(36.1)^{\mathrm{a}}$ & $132(48.9)^{b}$ & $39(54.2)^{\mathrm{b}}$ & \\
\hline \multicolumn{6}{|c|}{ Knowledge on diabetes-related complications, n (\%) } \\
\hline \multicolumn{2}{|c|}{ Yes } & $194(80.5)^{\mathrm{a}}$ & $246(91.1)^{\mathrm{b}}$ & $64(88.9)^{a . b}$ & \multirow{2}{*}{0.002} \\
\hline \multicolumn{2}{|l|}{ No } & $47(19.5)^{\mathrm{a}}$ & $24(8.9)^{\mathrm{b}}$ & $8(11.1)^{a . b}$ & \\
\hline \multirow[b]{2}{*}{ Retinopathy } & No & $72(29.9)^{a}$ & $33(12.2)^{\mathrm{b}}$ & $19(26.4)^{\mathrm{a}}$ & \multirow{2}{*}{$<0.001$} \\
\hline & Yes & $169(70.1)^{\mathrm{a}}$ & $237(87.8)^{\mathrm{b}}$ & $53(73.6)^{\mathrm{a}}$ & \\
\hline \multirow[b]{2}{*}{ Nephropathy } & No & $84(34.9)^{a}$ & $42(15.6)^{\mathrm{b}}$ & $20(27.8)^{a . b}$ & \multirow{2}{*}{$<0.001$} \\
\hline & Yes & $157(65.1)^{\mathrm{a}}$ & $228(84.4)^{\mathrm{b}}$ & $52(72.2)^{a . b}$ & \\
\hline \multirow[b]{2}{*}{ Neuropathy } & No & $159(66)^{\mathrm{a}}$ & $124(45.9)^{b}$ & $39(54.2)^{a . b}$ & \multirow{2}{*}{$<0.001$} \\
\hline & Yes & $82(34)^{a}$ & $146(54.1)^{\mathrm{b}}$ & $33(45.8)^{a . b}$ & \\
\hline \multirow[b]{2}{*}{ CVD } & No & $150(62.2)^{\mathrm{a}}$ & $94(34.8)^{\mathrm{b}}$ & $30(41.7)^{\mathrm{b}}$ & \multirow{2}{*}{$<0.001$} \\
\hline & Yes & $91(37.8)^{\mathrm{a}}$ & $176(65.2)^{\mathrm{b}}$ & $42(58.3)^{b}$ & \\
\hline \multirow[b]{2}{*}{ Sexual dysfunction } & No & $191(79.3)^{\mathrm{a}}$ & $185(68.5)^{b}$ & $49(68.1)^{\mathrm{b}}$ & \multirow{2}{*}{0.015} \\
\hline & Yes & $50(20.7)^{\mathrm{a}}$ & $85(31.5)^{b}$ & $23(31.9)^{\mathrm{b}}$ & \\
\hline
\end{tabular}

CVD: Cardiovascular disease; OADs: Oral antidiabetic agents. Pearson chi-square test. For the variables in the same row, statistically significant difference exists only between those marked with different lowercase letter 


\subsection{Knowledge on hypoglycemia symptoms and diabetes-related complications according to anti-diabetic treatments}

Percentage of patients knowing the overall hypoglycemia symptoms $(93.0 \%$ vs. $84.6 \%$, $p=0.010$, heavy sweating in particular) and diabetes-related complications ( $91.1 \%$ vs. $80.5 \%, \mathrm{p}=0.002$, nephropathy and neuropathy, in particular) were significantly higher in the OADs + insulin group compared to the OADs group (Table 5).

$\mathrm{OAD}$ therapy was associated with lowest rates of knowing blurred vision $(\mathrm{p}=0.004)$ and difficulty concentrating $(\mathrm{p}=0.003)$ as hypoglycemia symptoms as well as CVD $(\mathrm{p}<0.001)$ and sexual dysfunction $(\mathrm{p}=0.015)$ as complications of diabetes, while $\mathrm{OAD}+$ insulin therapy was associated with highest rate of knowing retinopathy $(\mathrm{p}<0.001)$ (Table 5).

\section{Discussion}

Our findings revealed high prevalence of poor glycemic control and poor diabetes self-care practice in T2D patients along with low rates of previous diabetes education and insufficient disease knowledge particularly in terms of definition and target levels of HbA1c rather than hypoglycemia symptoms and diabetes-related complications. The lower likelihood of knowing definition and target levels of $\mathrm{HbA} 1 \mathrm{c}$ and diabetes-related complications was noted in older patients ( $>45$ years), and those with lower educational attainment and lower income, while knowing hypoglycemia symptoms and diabetes-related complications were less common among insulin-naïve patients. The self-care practice regarding exercise and adherence to treatment was also poorer in patients with lower educational level, whereas no significant difference was noted in SMBG practice according to educational attainment, patient age or income class.

HbA1c levels were $>8 \%$ in half of our patients despite ongoing OADs \pm insulin treatment along with lack of diabetes education and insufficient knowledge on HbA1c targets in almost half of patients. The rate for poor glycemic control in the current study is consistent with current estimates on failure to achieve or sustain the recommended HbA1c target of $<7.0 \%$ in almost $50 \%$ of diabetic patients, despite novel therapeutics [1,2,20]. Likewise, poor general knowledge on diabetes has consistently been reported in studies with diabetic patients across the world [11,21-23], despite its significant adverse effect on self-care practice, adherence to medications and thus on clinical outcome $[11,22,24,25]$.

In the current study, disease knowledge was favorable regarding the hypoglycemia symptoms and diabetes-related complications, whereas lack of self-knowledge on the definition and target levels of HbA1c was evident in more than half of the study population. High awareness of hypoglycemia symptoms in our cohort, particular by insulin-treated patients, seems notable given association of the impaired vs. normal awareness of hypoglycemia with experiencing a higher incidence of severe hypoglycemia [26]. Insufficient knowledge on HbA1c in our cohort supports the data from GUIDANCE study, indicated self-knowledge on HbA1c only in half of the patients, despite association of HbA1c selfknowledge with a better glycemic control [27].

Nonetheless, the disease knowledge regarding HbA1c, diabetes-related complications and specific hypoglycemic symptoms was poorer among insulin-naïve patients, older patients and in patients with lower educational level and lower monthly income. Likewise, positive correlations of diabetes knowledge and practice was reported with academic education, monthly income and diabetes education along with importance of diabetes knowledge in enabling improved self-care practices and thus better adaptability of disease and receptivity of therapy [8,28-32]. While the studies revealed inconsistent findings on the association of patient age with diabetes self-care practice or disease knowledge, some studies reported poorer self-care practice and poorer disease knowledge in older T2D patients along with a 3\% decrease in diabetes knowledge score with every ten years increase in age [8,33-36].

Notably, while most of patients reported that they know hypoglycemia symptoms and diabetes-related complications overall, the difficulty concentrating and sexual dysfunction were the least commonly reported hypoglycemia symptom and diabetes-related complication by our T2D patients, respectively. In addition, lower knowledge levels on difficulty concentrating and sexual dysfunction were more common among insulin-naïve, older age patients and those with lower educational and income levels.

The identification of sexual dysfunction as the least commonly rated diabetes-related, particularly by older patients and those with lower educational level and lowest income seems notable given the high prevalence of sexual inactivity and related sexual distress reported among middle-aged and older men and women with T2D [37]. Moreover, in a systematic review of 28 studies on the impact of socioeconomic status on diabetes complications, low levels of education or income was reported to be associated with increased risk of retinopathy, nephropathy, neuropathy and CVD [13]. 
Hence, given the possibility of T2D patients in the lowest socioeconomic levels to experience greater risks of microvascular and macrovascular diabetes complications, authors suggested that socioeconomic status, as a new and important risk factor for poor outcomes, to be integrated into the clinical evaluation of T2D patients [13].

Our findings indicate a need for improved diabetes self-care practice in terms of regular medication usage, SMBG and regular exercise, given the high rates of poor self-care practice reported by half to two-thirds of our patients. This supports the well-documented suboptimal adherence to self-management among diabetic patients [38], despite the fact that self-care practice, particularly SMBG, is considered a cornerstone of diabetes care enabling patient participation in achieving and maintaining specific glycemic targets [39]. Incorporation of SMBG into daily routine and more often testing (at least twice a day) have been considered to be associated with improved glycemic control in diabetic patients by providing information about current glycemic status and thus guiding adjustments in diet, exercise and medication $[12,39,40]$.

In fact, poor SMBG practice was prevalent among T2D patients in our cohort, regardless of patient age, educational attainment and income class, whereas knowing definition and target levels of HbA1c was more likely with younger patient age, higher educational attainment and higher income class. Hence our findings emphasize the impact of patient age, educational attainment and income class on having disease knowledge but not on implementing an appropriate SMBG practice, supporting the consideration of a discrepancy between theory and practice in T2D patients with continuation of poor self-care practice despite having good disease knowledge scores [7].

Nonetheless, given that $20 \%$ of patients in the current study were suffering from diabetes less than 5 years, the poor SMBG practice regardless of sociodemographic background in the current study may also emphasize the potential impact of diabetes duration, since diabetes self-care practice may be less carefully followed in the early stages of disease and may improve only after emergence of diabetes-related complications. Notably, disease duration of $<6$ years, lack of SMBG and insufficient knowledge on diabetes have been reported to be significant determinants of poor self-care practices among diabetic patients [40] along with positive impact of disease duration on the diabetes self-care practice $[41,42]$.

In addition, the lower disease knowledge among insulin-naïve patients on OAD therapy in the current study seems also emphasize the likelihood of improved diabetes self-care with initiation of insulin in T2D patients due to increased awareness of the seriousness of the disease and emergence of related concerns among patients that interferes with their self-management efforts [43,44].

Poor self-care practice in our cohort seems also notable given the lack of diabetes education and non-adherence to dietary recommendations despite taking a dietary education class noted in nearly half of our patients. Hence, our findings support the consideration of diabetes education to be an underutilized measure of diabetes care with low rates of active participation in diabetes education among diabetic population, despite its association with improved glycemic control and better adaptation to treatment and changes in lifestyle $[10,12,45,46]$.

Formal education is considered likely to be especially helpful in diabetes in terms of dealing with complex self-managed treatment regimens [15]. Hence, poor academic education is considered to jeopardize the ability of patients to comprehend, retain, and enact essential self-care behaviors [15,47]. Notably, in the current study lower educational attainment was associated with poor disease knowledge in terms of glycemic control, hypoglycemia symptoms and diabetes-related complications as well as with poor self-care practice in terms of regular exercise and adherence to antidiabetic treatment. This seems notable given that educational attainment is considered to moderate the success of diabetes education in terms of obtaining optimal glycemic control, even after controlling for household income $[15,48]$. Indeed, attending diabetes education class was reported to be positively associated with optimal glycemic control in diabetes patients with higher education rather than those with less than middle school education [15]. However, when an intensive patient education based on improved staff accessibility, encouragement, and support was implemented with frequent clinic visits and phone contact by staff, the greatest improvement in glycemic control was reported to be achieved in patients with less educational attainment and lower health literacy $[48,49]$. Hence, authors suggested the potential benefit of more intensive diabetes self-care education interventions with simpler protocols for patients with less formal education, while the likelihood of those with more formal education to be effectively treated by less intensive interventions $[15,17]$.

In addition, the association of older age with lower disease knowledge in our study supports the previously reported significant and independent association of age with post-diabetes education intervention knowledge, with older patients to learn significantly less than younger patients, despite their specific needs and increased health risks [19]. 
Hence, our findings support the potential role of adopting continuous practical and feasible educational interventions starting from the time of diagnosis in achievement of improved self-care practice and treatment adherence in diabetic patients $[39,41,43,50]$, while also emphasize the likelihood of higher efficacy of educational interventions when tailored to how people learn best in accordance with needs and cognitive capacities of the target population [15,19].

Notably, majority of our patients reported distance from home to hospital, waiting time in the hospital and quality of doctor-patient relationship to be favorable. However, in a narrative review, authors reported that while majority of T2D patients rates the quality of their relations with the health professionals as good, they also complain of a lack of specific and individualized attention and the authoritarian figure represented by doctors, particularly the younger patients and those with higher educational level [43].

Hence, due to potential differences in healthcare providers' communication with higher versus lower educated patients, the importance of enhanced communication between healthcare providers has consistently been emphasized along with the presence of healthcare systems that value patient-centered care and encourage doctors to engage patients in such dialogue $[17,51,52]$.

Certain limitations to this study should be considered. First, the qualitative cross-sectional study design and relatively small non-representative sample may limit our ability to make causal inferences as well as generalizability our findings. Second, lack of data on psychometric tools to assess diabetes knowledge, self-care behavior and medication adherence is another limitation which otherwise would extend the knowledge achieved in the current study.

In conclusion, our findings revealed poor glycemic control, low level of knowledge on definition and target levels of HbA1c and lack of diabetes education with suboptimal adherence to self-care practice regarding regular medication usage, SMBG, diet and regular exercise in a considerable percentage of T2D patients. Disease knowledge but not SBGM practice significantly differed with respect to patient age, educational attainment, income class and type of ongoing antidiabetic treatment. Accordingly our findings seem to indicate lower disease-related insight among older patients and those with lower educational and income levels, emphasizing the potential role of individualized diabetes education interventions specifically designed with careful choice of method and contact time and tailored to needs of patients in accordance with age, educational level and socioeconomic background to improve disease knowledge and thus the adherence to self-care practice in T2D patients. The self-care practice and disease knowledge should be further addressed with respect to educational and socioeconomic background of target population by larger scale qualitative studies to better understand the effectiveness of diabetes prevention treatments in diverse populations.

\section{Conclusion}

In conclusion, our findings revealed poor glycemic control, low level of knowledge on definition and targets of HbA1c and lack of diabetes education with suboptimal adherence to self-care practice in a considerable percentage of patients. Disease knowledge but not SMBG practice significantly differed with respect to patient age, educational attainment, income class and treatment. Our findings seem to indicate lower disease-related insight among older patients and those with lower educational and income levels, emphasizing the potential role of individualized diabetes education interventions tailored to needs of patients to improve disease knowledge and thus the adherence to self-care practice in T2D patients.

\section{Compliance with ethical standards}

\section{Acknowledgments}

The authors acknowledge the nurses and physicians who interviewed face to face with diabetic patients.

\section{Disclosure of conflict of interest}

There is no conflict of interest among the authors or any person.

\section{Statement of ethical approval}

This study was conducted in accordance with the ethical principles stated in the "Declaration of Helsinki" and approved by the institutional ethics committee. 


\section{Statement of informed consent}

Written informed consent was obtained from all individual participants included in this study.

\section{References}

[1] Carls G, Huynh J, Tuttle E, Yee J, Edelman SV. Achievement of glycated hemoglobin goals in the US remains unchanged through 2014. Diabetes Ther. 2017; 8: 863-73.

[2] Stone MA, Charpentier G, Doggen K, Kuss O, Lindblad U, Kellner C, et al.; GUIDANCE Study Group. Quality of care of people with type 2 diabetes in eight European countries. Diabetes Care. 2013; 36: 2628-38.

[3] Effect of intensive blood-glucose control with metformin on complications in overweight patients with type 2 diabetes (UKPDS 34). UK Prospective Diabetes Study (UKPDS) Group. Lancet. 1998; 352: 854-65.

[4] Davies MJ, D’Alessio DA, Fradkin J, Kernan WN, Mathieu C, Mingrone G, et al. Management of Hyperglycemia in Type 2 Diabetes, 2018. A Consensus Report by the American Diabetes Association (ADA) and the European Association for the Study of Diabetes (EASD). Diabetes care. Dec 2018; 41(12): 2669-2701.

[5] Beck J, Greenwood DA, Blanton L, Bollinger ST, Butcher MK, Condon JE, Cypress M, Faulkner P, Fischl AH, Francis T, Kolb LE, Lavin-Tompkins JM, MacLeod J, Maryniuk M, Mensing C, Orzeck EA, Pope DD, Pulizzi JL, Reed AA, Rhinehart AS, Siminerio L, Wang J; 2017 Standards Revision Task Force. 2017 National Standards for Diabetes Self-Management Education and Support. Diabetes Educ. Oct 2017; 43(5): 449-464.

[6] Powers MA, Bardsley J, Cypress M, et al. Diabetes Self-management Education and Support in Type 2 Diabetes: A Joint Position Statement of the American Diabetes Association, the American Association of Diabetes Educators, and the Academy of Nutrition and Dietetics. Clin Diabetes. 2016; 34(2): 70-80.

[7] Abdulrahman M, Husain ZSM, Abdouli KA, Kazim MN, Sayed Mahdi Ahmad F, Carrick FR. Association between knowledge, awareness, and practice of patients with type 2 diabetes with socio-economic status, adherence to medication and disease complications. Diabetes Res Clin Pract. 2020 May 01; 163: Article ID 108124.

[8] Abbasi YF, See OG, Ping NY, Balasubramanian GP, Hoon YC, Paruchuri S. Diabetes knowledge, attitude, and practice among type 2 diabetes mellitus patients in Kuala Muda District, Malaysia - A cross-sectional study. Diabetes Metab Syndr. 2018; 12(6): 1057-1063.

[9] Asmelash D, Abdu N, Tefera S, Baynes HW, Derbew C. Knowledge, Attitude, and Practice towards Glycemic Control and Its Associated Factors among Diabetes Mellitus Patients. J Diabetes Res. 8 Apr 2019; Article ID 2593684, 9 pages.

[10] Chrvala CA, Sherr D, Lipman RD. Diabetes self-management education for adults with type 2 diabetes mellitus: A systematic review of the effect on glycemic control. Patient Educ Couns. Jun 2016; 99(6): 926-4.

[11] Kassahun T, Gesesew H, Mwanri L, Eshetie T. Diabetes related knowledge, self-care behaviours and adherence to medications among diabetic patients in Southwest Ethiopia: a cross-sectional survey. BMC Endocr Disord. 31 May 2016; 16(1): 28.

[12] Weller SC, Baer R, Nash A, Perez N. Discovering successful strategies for diabetic self-management: a qualitative comparative study. BMJ Open Diabetes Res Care. 8 May 2017; 5(1): e000349.

[13] Tatulashvili S, Fagherazzi G, Dow C, Cohen R, Fosse S, Bihan H. Socioeconomic inequalities and type 2 diabetes complications: A systematic review. Diabetes Metab. Apr 2020; 46(2): 89-99.

[14] Chaturvedi N, Stephenson JM, Fuller JH. The relationship between socioeconomic status and diabetes control and complications in the EURODIAB IDDM complications study. Diabetes Care. 1996; 19: 423-30.

[15] Sacco WP, Bykowski CA, Mayhew LL, White KE. Educational attainment moderates the effect of a brief diabetes self-care intervention. Diabetes Res Clin Pract. Jan 2012; 95(1): 62-7.

[16] Redmond EH, Burnett SM, Johnson MA, Park S, Fischer JG, Johnson T. Improvement in A1C levels and diabetes self-management activities following a nutrition and diabetes education program in older adults. J Nutr Elder. 2007; 8: 3-102.

[17] O'Brien MJ, Whitaker RC, Yu D, Ackermann RT. The comparative efficacy of lifestyle intervention and metformin by educational attainment in the Diabetes Prevention Program. Prev Med. 2015; 77: 125-130. 
[18] Hessler DM, Fisher L, Mullan JT, Glasgow RE, Masharani U. Patient age: a neglected factor when considering disease management in adults with type 2 diabetes. Patient Educ Couns. 2011; 85(2): 154-159.

[19] Ghisi GLM, Aultman C, Konidis R, et al. Effectiveness of an education intervention associated with an exercise program in improving disease-related knowledge and health behaviours among diabetes patients [published online ahead of print, 2020 Apr 21]. Patient Educ Couns. 2020 Sep; 103(9): 1790-1797.

[20] American Diabetes Association, Foundations of care: education, nutrition, physical activity, smoking cessation, psychosocial care, and immunization, Diabetes Care. 2015; 38: S20-S30.

[21] Shah VN, Kamdar PK, Shah N. Assessing the knowledge, attitudes and practice of type 2 diabetes among patients of Saurashtra region, Gujarat. Int J Diabetes Dev Ctries. 2009; 29: 118-22.

[22] Al-Maskari F, El-Sadig M, Al-Kaabi JM, Afandi B, Nagelkerke N, Yeatts KB. Knowledge, attitude and practices of diabetic patients in the United Arab Emirates. PLoS One. 2013; 8(1): e52857.

[23] Siddique MKB, Islam SMS, Banik PC, Rawal LB. Diabetes knowledge and utilization of healthcare services among patients with type 2 diabetes mellitus in Dhaka, Bangladesh. BMC Health Serv Res. 22 Aug 2017; $17(1): 586$.

[24] Islam SM, Niessen LW, Seissler J, Ferrari U, Biswas T, Islam A, Lechner A. Diabetes knowledge and glycemic control among patients with type 2 diabetes in Bangladesh. Springer Plus. 2015; 4: 284.

[25] Feleke SA, Alemayehu CM, Adane HT. Assessment of the level and associated factors with knowledge and practice of diabetes mellitus among diabetic patients attending at FelegeHiwot hospital, Northwest Ethiopia. Clinical and Medicine Research. 2013; 2(6): 110-20.

[26] Henderson JN, Allen KV, Deary IJ, Frier BM. Hypoglycaemia in insulin-treated Type 2 diabetes: frequency, symptoms and impaired awareness. Diabet Med. 2003; 20(12): 1016-21.

[27] Trivedi H, Gray LJ, Seidu S, Davies MJ, Charpentier G, Lindblad U, et al. Self-knowledge of HbA1c in people with Type 2 Diabetes Mellitus and its association with glycaemic control. Prim Care Diabetes. Oct 2017; 11(5): 414420.

[28] Zainudin SB, Ang DY, Soh AW. Knowledge of diabetes mellitus and safe practices during Ramadan fasting among Muslim patients with diabetes mellitus in Singapore. Singapore Med J. 2017; 58(5): 246-52.

[29] Kassahun CW, Mekonen AG. Knowledge, attitude, practices and their associated factors towards diabetes mellitus among non-diabetes community members of Bale zone administrative towns, South East Ethiopia. A crosssectional study. PLoS One. 2017; 12(2): e0170040.

[30] Van der Heide I, Uiters E, Rademakers J, Struijs JN, Schuit AJ, Baan CA. Associations among health literacy, diabetes knowledge, and selfmanagement behavior in adults with diabetes: results of a dutch crosssectional study. J Health Commun. 2014; 19(2): 115-31.

[31] Kueh YC, Morris T, Ismail A-A-S. The effect of diabetes knowledge and attitudes on self-management and quality of life among people with type 2 diabetes. Psychol Health Med. 2017; 22(2): 138-44.

[32] Steed L, Cooke D, Newman S. A systematic review of psychosocial outcomes following education, selfmanagement and psychological interventions in diabetes mellitus. Patient Educ Couns. 2003; 51(1): 5-15.

[33] Niroomand M, Ghasemi SN, Karimi-Sari H, Kazempour-Ardebili S, Amiri P, Khosravi MH. Diabetes knowledge, attitude and practice (KAP) study among Iranian in-patients with type-2 diabetes: a cross-sectional study. Diabetes Metab Syndr. 2016; 10(1): S114-119.

[34] West JD, Goldberg KL. Diabetes self-care knowledge among outpatients at a veteran's affairs medical center. Am J Health Syst Pharm. 2002; 59(9): 849-52.

[35] Islam FM, Chakrabarti R, Dirani M, Islam MT, Ormsby G, Wahab M, et al. Knowledge, attitudes and practice of diabetes in rural Bangladesh: the Bangladesh population based diabetes and eye study. PLOS ONE. 2014; 9: e110368.

[36] Garrison RJ, Gold RS, Wilson PW, Kannel WB. Educational attainment and coronary heart disease risk: the Framingham Offspring Study. Prev Med. 1993; 22: 54-64.

[37] Bjerggaard M, Charles M, Kristensen E, Lauritzen T, Sandbæk A, Giraldi A. Prevalence of Sexual Concerns and Sexual Dysfunction among Sexually Active and Inactive Men and Women with Screen-Detected Type 2 Diabetes. Sex Med. 2015; 3(4): 302-310. 
[38] Carpenter R, DiChiacchio T, Barker K. Interventions for self-management of type 2 diabetes: An integrative review. Int J Nurs Sci. 14 Dec 2018; 6(1): 70-91.

[39] Shrivastava SR, Shrivastava PS, Ramasamy J. Role of self-care in management of diabetes mellitus. J Diabetes Metab Disord. 2013; 12: 14.

[40] Dedefo MG1, Ejeta BM2, Wakjira GB3, Mekonen GF3, Labata BG3. Self-care practices regarding diabetes among diabetic patients in West Ethiopia. BMC Res Notes. 8 Apr 2019; 12(1): 212.

[41] Alshammari ZJ1, Alsaid LA1, Parameaswari PJ2, Alzahrani AA3. Attitude and knowledge about foot care among diabetic patients in Riyadh, Saudi Arabia. J Family Med Prim Care. Jun 2019; 8(6): 2089-2094.

[42] Yılmaz Karadă̆ F, Saltoğlu N, Ak Ö, et al. Foot self-care in diabetes mellitus: Evaluation of patient awareness. Prim Care Diabetes. 2019; 13(6): 515-520.

[43] Nombela Manzaneque N, Pérez-Arechaederra D, Caperos Montalbán JM. Side effects and practices to improve management of type 2 diabetes mellitus from the viewpoint of patient experience and health care management. A narrative review. Endocrinol Diabetes Nutr. 2019; 66(10): 596-610.

[44] Mosnier-Pudar H, Hochberg G, Eschwege E, Virally ML, HalimiS, Guillausseau PJ, et al. How do patients with type 2 diabetesperceive their disease? Insights from the French DIABASIS survey.Diabetes Metab. 2009; 35: 220-7.

[45] Norris SL, Engelgau MM, Narayan K. Effectiveness of self-management training in type 2 diabetes: a systematic review of randomized controlled trials, Diabetes Care. 2001; 24: 561-587.

[46] Norris SL, Lau J, Smith SJ, Schmid CH, Engelgau MM. Self-management education for adults with type 2 diabetes: a meta-analysis of the effect on glycemic control, Diabetes Care. 2002; 25: 1159-1171.

[47] Peters E, Baker DP, Dieckmann NF, Leon J, Collins J. Explaining the effect of education on health: a field study in Ghana. Psychol Sci. 2010; 21(10): 1369-1376.

[48] Goldman DP, Smith JP. Can patient self-management help explain the SES health gradient? Proc Natl Acad Sci USA. 2002; 99: 10929-34.

[49] Rothman RL, DeWalt DA, Malone R, Bryant B, Shintani A, Crigler B, et al. Influence of patient literacy on the effectiveness of a primary care-based diabetes disease management program. JAMA. 2004; 1711-6.

[50] Glasgow RE, Strycker LA. Preventive care practices for diabetes management in two primary care samples. Am J Prev Med. 2000; 19(1): 9-14.

[51] Entwistle VA, Watt IS. Treating patients as persons: a capabilities approach to support delivery of personcentered care. Am J Bioeth. 2013; 13(8): 29-39.

[52] Sabee CM, Koenig CJ, Wingard L, et al. The Process of Interactional Sensitivity Coding in Health Care: Conceptually and Operationally Defining Patient-Centered Communication. J Health Commun. 2015; 20(7): 773-782. 